Noname manuscript No.

(will be inserted by the editor)

Stefano Bernardi . J. S. Hansen - Federico

Frascoli · B. D. Todd · Carl P. Dettmann

\title{
Ergodicity of a single particle confined in a nanopore
}

August 23, 2012

\begin{abstract}
We analyze the dynamics of a gas particle moving through a nanopore of adjustable width with particular emphasis on ergodicity. We give a measure of the portion of phase space that is characterized by quasiperiodic trajectories which break ergodicity. The interactions between particle and wall atoms are mediated by a Lennard-Jones potential, so that an analytical treatment of the dynamics is not feasible, but making the system more physically realistic. In view of recent studies, which proved non-ergodicity for systems with scatterers interacting via smooth potentials, we find that the non-ergodic component of the phase space for energy levels typical of experiments, is surprisingly small, i.e. we conclude that the ergodic hypothesis is a reasonable approximation even for a single particle trapped in a nanopore. Due to the numerical scope of this work, our focus will be the onset of ergodic behaviour which is evident on time scales accessible to simulations and experimental observations rather than ergodicity in the infinite time limit.
\end{abstract}

Keywords Ergodic theory · statistical mechanics · dynamical systems · Lyapunov exponents

Stefano Bernardi

Australian Institute for Bioengineering \& Nanotechnology,

The University of Queensland, QLD 4072, Australia

E-mail: s.bernardi1@uq.edu.au

J. S. Hansen

DNRF Centre "Glass and Time",

IMFUFA, Department of Sciences, Roskilde University, DK-4000 Roskilde, Denmark

E-mail: jschmidt@ruc.dk

Federico Frascoli

Department of Mathematics and Statistics,

University of Melbourne, Parkville, Victoria 3010, Australia

B. D. Todd

Mathematics, Faculty of Engineering \& Industrial Sciences and Centre for Molecular Simulation, Swinburne University of Technology, Hawthorn, Victoria 3122, Australia

Carl P. Dettmann

School of Mathematics,

University of Bristol, Bristol BS8 1TW, United Kingdom 


\section{Introduction}

The ergodic hypothesis equates the long time average of a physical observable $B$ to its ensemble average [1], which means that a phase space trajectory will spend in each phase space volume of equal probability (measure), an equal amount of time. This can be written as

$$
\lim _{t \rightarrow \infty} \frac{1}{t} \int_{0}^{t} B\left(\boldsymbol{\Gamma}\left(t^{\prime}\right)\right) d t^{\prime}=\int_{\boldsymbol{\Gamma}} B(\boldsymbol{\Gamma}) f(\boldsymbol{\Gamma}) d \boldsymbol{\Gamma},
$$

where $\boldsymbol{\Gamma}(t)=[\mathbf{r}(t), \mathbf{p}(t)]^{T}$ is a point in the phase space at time $t$. Alternatively we can say that, given the phase-space domain $\mathrm{X}$ of a system, the transformation that generates its dynamics is ergodic with respect to the invariant measure $d \mu=f(\boldsymbol{\Gamma}) d \boldsymbol{\Gamma}$ if, for all measurable invariant sets $A \subset X$, either $\mu(A)=0$ or $\mu(X \backslash A)=0[2,3]$.

Even though it is known that most systems of physical interest violate ergodicity, it is still assumed valid for practical purposes when computing phase variables of interest such as temperature and pressure. This is also the case for systems which are away from the thermodynamic limit or which are not strictly thermodynamic, i.e. the physical properties depend on the geometry of the system boundary [4].

Many studies have been done on ergodicity of hard spheres trapped in billiards [5-7] or moving around periodic array of scatterers. Varying the nature of the scatterers i.e. spherical for a Lorentz gas $[8,9]$ or square for Ehrenfest wind-tree model [10] and the boundary conditions $[11,12]$, chaotic motion and a range of transport properties can be observed. The appeal of these systems rests on the possibility of an analytical treatment, which may lead to definitive proofs, something rarely possible in systems with soft potentials.

In the last few decades progress has been done in the treatment of generalized Sinai billiards $[11,13]$ which parametrize the interactions mediated by steep and smooth potentials. In these systems, one accounts for the portion of a particle's trajectory which is curved by the action of the scatterer's potential (at distances shorter than the interaction cutoff), by introducing an angle of rotation around the scatterer itself. Typically, to prove the occurrence of ergodicity-breaking, a periodic orbit is identified in the system, it is then proven to be elliptic due to the focusing effect of the potential under proper conditions, and finally invoking the non-degeneracy condition of KAM theory [14], it is proven to be stable (i.e. the KAM tori have non-zero measure).

Other studies, similar in essence, looked at systems with steep repulsive potentials, where the geometrical displacement of the scatterers allowed their potential clouds to overlap and form a corner at the meeting point $[15,16]$. These corners, named corner polygons, occur for example along atomic wall layers at nanochannel boundaries. Studying the stability of the return map for a periodic orbit it is possible to prove ergodicity-breaking. These studies focus on the local structures which give rise to such behaviour, but they do not provide information on how relevant non-ergodicity is when these configurations are embedded in more extended systems. Also the complete or partial extension of these results to encompass a more broader class of geometries might prove difficult, and in these cases a numerical approach is needed. Recent works have also investigated the connection between the dynamics at the microscopic level and macroscopic transport properties, in particular how transport takes place in chaotic and non-chaotic systems $[17,18]$, or the identification of several transport regimes (e.g. diffusive, superdiffusive, etc.) in polygonal channels [19].

An alternative approach proposed by Khinchin [3] avoids invoking ergodic theorems to equate time and ensemble averages. In his argument, Khinchin suggests that one can, for a special class of functions (sum-functions), approximate the phase average to the time average to a given accuracy in the thermodynamic limit. Loosely speaking, because sum-functions depend on the sum of functions of single phase space coordinates, they assume the expected value almost everywhere when the number of degrees of freedom is large. However, when one deals with small systems, e.g. a nanopore as in the present work, and therefore characterized by few degrees of freedom, 
sum-functions can show large fluctuations from the expected value and Kinchin's argument will not apply.

When forces are included in the models and the bounding geometry becomes irregular the answer to the fundamental question of ergodicity becomes elusive. Even for the simple system considered herein, the amount of time required to follow the gas particle while wandering the whole phase space is inaccessible to simulations. We therefore focus on the short time scales accessible to simulations in order to investigate if a violation of ergodicity occurs. Many studies of practical interest, in fact, look at properties (e.g. single particle or molecule diffusion in nanopores [20,21] or pattern recognition in nanochannels [22]) which reveal themselves on these time scales and which are naturally connected to the ergodic hypothesis. This work will also be relevant for these short time experiments.

We shall here characterize the dynamical properties of a simple system, focusing in particular on ergodicity. We study a single particle trapped in a nanopore and, by letting the particle interact with the atomistic walls through a soft potential, we try to retain as much details as possible in order to improve the connection with real models. Since the system under study is composed of soft disks (wall particles and gas particle), one could expect that for high enough energies, the defocusing nature of the collisions with the walls would dominate and, as in dispersing billiards [23], quickly bring the particle to wander the whole phase space available. However, as we will show, this is not always the case. In fact, the areas where the ergodic hypothesis is not valid are scattered throughout the entire phase space.

\section{Model}

We will now describe the model in detail. A particle moving in two dimensions, with phase space variables $\mathbf{r}(t)=(x(t), y(t))$ and $\mathbf{p}(t)=\left(p_{x}(t), p_{y}(t)\right)$, is confined between two perfectly rigid atomistic walls, where the position of each wall atom is kept fixed at all times. The equations of motion for the system are obtained from its Hamiltionian [24], $H: \mathbb{R}^{4} \rightarrow \mathbb{R}$, which reads

$$
H(\mathbf{r}, \mathbf{p})=\frac{\mathbf{p}^{2}}{2 m}+\sum_{i=1}^{N_{w}} U\left(\left|\mathbf{r}-\mathbf{r}_{i}\right|\right)
$$

Here $m$ is the particle mass, $N_{w}$ is the number of wall particles and $U\left(\left|\mathbf{r}-\mathbf{r}_{i}\right|\right)$ is the potential (or configurational) contribution to the total energy, $\mathbf{r}_{i}$ being the position vector of the $i$ th wall atom. The functional form of $U$ is given by the truncated and shifted Lennard-Jones potential [25]

$$
U\left(\left|\mathbf{r}-\mathbf{r}_{i}\right|\right)=U\left(r_{i}\right)=4 \epsilon\left[\left(\frac{\sigma}{r_{i}}\right)^{12}-\left(\frac{\sigma}{r_{i}}\right)^{6}\right]-U\left(r_{c}\right),
$$

where $\epsilon$ and $\sigma$ define interaction and length scale, respectively, $r_{i}=\left|\mathbf{r}-\mathbf{r}_{i}\right|$ is the distance between the particle and the $i$ th wall atom and $r_{c}=2.5 \sigma$. Any quantity is expressed in reduced units of $m$, $\epsilon$ and $\sigma$ with $m=\epsilon=\sigma=1$, see Ref. [25], and we will, as is common practice, omit writing these units explicitly throughout the paper. Each wall consists of two atomic layers which are separated by $\sqrt{3} x_{0}$, with $x_{0}=0.5557$, as illustrated in Fig. 1 . The layers facing the channel are separated by $2 y_{0}$, where $y_{0}$ represent a control parameter that can be varied, and in each wall layer the distance in the $x$ direction between the atoms is set to $2 x_{0}$. This arrangement ensures that the walls are impenetrable and has a direct effect on the shape of periodic oscillations displayed by the system as we will see later. The truncation ensures that, for any position of the particle and for any channel width, the force exerted on the particle is restricted to the interactions between the particle and no more than six wall particles. Also, note that the system is periodic in the $x$ direction and $N_{w}=20$ are used to avoid any size effect due to the periodic boundary conditions. 
The system is non-integrable [26], in the sense that only the Hamiltonian is a constant of motion, the dynamics is confined to a three dimensional hypersurface and chaotic dynamics can be present [26]. It is possible to rewrite the governing differential equations such that the phase space is three dimensional, however, we will simply use Hamiltonian equations of motion since they have a clear physical interpretation. Standard molecular dynamics (MD) simulations were used to generate the dynamics, where the equations of motion were integrated forward in time using a second-order symplectic leap-frog integration scheme [27], with time step $\Delta t=0.002$.

\section{Results and Discussion}

\subsection{Basic analysis of the dynamics}

The potential energy surface provides information about the dynamics and the locations of the fixed points: from the Hamiltonian equations of motion, it can be seen that at any fixed point the particle has a zero momentum, $\mathbf{p}(t)=\mathbf{0}$, hence they are given by $\left(\mathbf{r}_{s}, \mathbf{0}\right)$, where $\mathbf{r}_{s}=\left(x_{s}, y_{s}\right)$ is found from the extrema of $U$. This in turn means that the fixed points are given by the zeros of the force $\mathbf{F}: \mathbb{R}^{2} \rightarrow \mathbb{R}^{2}$. Sections of the contour plots for the potential energy surfaces, $U: \mathbb{R}^{2} \rightarrow \mathbb{R}$, for four different channel widths, are shown in Fig. 2. The regions surrounding the wall atoms where the potential energy increases dramatically are indicated by the white areas.

The crosses represents saddle points and the circles center points: these are the only two types of fixed points that can exist [26]. Given the geometry of the channel, these always occur along two fixed direction at constant $x$, with coordinates $x_{s 1}=2 n x_{0}$ and $x_{s 2}=(2 n+1) x_{0}$ with $n=0,1,2, \ldots$ (see Fig. 1 ). To determine which channel widths to investigate, a one parameter bifurcation analysis was carried out using the width $y_{0}$ as the continuation parameter. For a full account of the procedure, the reader is referred to Ref. [28]. For reasons of brevity, suffice it to say that a term can be added to the equations of motion and that this term is a function of the first integrals of Eq. 2. This addition transforms the system into a dissipative one, and allows to find changes in the extrema as the parameter $y_{0}$ varies. Thus, by looking at the bifurcations that occur along the two directions $x_{s 1}$ and $x_{s 2}$, regions of interest can be identified. The bifurcation plots for both $x_{s 1}$ and $x_{s 2}$ are clearly pitchfork diagrams, which, given their simplicity, are not shown. This can also be inferred by looking at Fig. 2, where, for example, the direction $x_{s 1}$ shows first one center at low $y_{0}=1.1$ (figure d), then it bifurcates into three points (pitchfork) for larger widths $y_{0}$ (figures $\mathrm{c}, \mathrm{b}$ and a). A similar fate happens for points belonging to $x_{s 2}$. Essentially, considering the bifurcations that occur along the $x_{s 1}$ and $x_{s 2}$ directions, we have chosen to investigate the ergodicity of the system for values $y 0=1.1,1.21,1.228$ and 1.4 , since they capture the relevant variations for the fixed points in the channel in line with the bifurcation analysis.

For example, if the initial conditions are given as $(x(0), y(0))=\left(2 n x_{0}, y(0)\right)$ and $\left(p_{x}(0), p_{y}(0)\right)=$ $\left(0, p_{y}(0)\right)$ the dynamics is analogous to the unforced frictionless Duffing oscillator [29] due to the symmetry of the system. Also, if the system possesses sufficiently low energy the particle may perform a periodic motion in one of the potential energy wells surrounding one of the center points in the secondary sections (see Fig. 2). To study the phase space trajectories further, Poincaré sections have been investigated for energies $E \leq-2$, i.e. in the regime where the particle is confined to one of the potential wells. In Fig. 3 we plot a Poincaré section in the $\left(x(t), p_{x}(t)\right)$ plane for $y=\sqrt{3} x_{0}+1$ and $E=-2$, where we observe dense, confined and chaotic regions. The Poincaré sections are symmetric about $x \approx 2 x_{0}$ and $p_{x}=0.0$ and confined to a relatively small region as is expected in the low energy regime. We have only included a single initial condition as well as excluded every second intersection for clarity: the full projection can simply be generated by symmetry considerations. The island chain [26] in Fig. 3 survives at $E=-1.9$ but is destroyed as $E$ increases further: for $E \geq-1.5$ the chaotic region fills out the entire region leaving no distinguishable structures. 
For these small energies we also expect the presence of KAM tori [26] as well as the chaotic regions that are limited between such tori. The existence of the tori for low energies is indicated in Fig. 4 a), where the power spectrum of the dynamical variable $y(t)$ is shown for $E=-2$. Two main frequencies, $f_{1}$ and $f_{2}$, are present with $f_{1}=1.6 \times 10^{4}$ and $f_{2}=1.14 \times 10^{4}$. The rotation number, $R=f_{1} / f_{2}$, is around 1.14, i.e. the particle undergoes quasiperiodic motion. For higher energies, Fig. $4 \mathrm{~b}$ ), an analysis of the power spectrum reveals large effects from a broad spectrum of frequencies which is a signature of chaotic motion.

The system is clearly not ergodic for such low energies, therefore in the analysis that will follow, we will only consider energies high enough to ensure that a particle is potentially able to travel through the pore without being trapped by the minima. We would like to note that the presence of trivial regular orbits is to be expected even for higher energies and different channel widths, due to the presence of symmetries in our system's geometry. However what is going to be relevant to this work is the stability (measure) of these and of other non-trivial islands.

\subsection{Characterization of Ergodicity and Chaos through Lyapunov Exponents}

In this section we carry out an analysis of the system's ergodicity through a study of the Lyapunov exponents for energies $E \geq-1$. Essentially, if small perturbations of initial conditions grow exponentially with time, the system exhibits chaos as quantified by one or more positive Lyapunov exponents. We would like to stress that the Lyapunov analysis presented in this work is only a means to an end, i.e. we are not trying to characterize the chaoticity of the channel as already done in previous works $[30,31]$, but only to determine and quantify its ergodicity using the Lyapunov exponents as a tool.

We can write the equations of motion in the form

$$
\dot{\Gamma}=\mathbf{G}(\boldsymbol{\Gamma}, t) .
$$

The evolution of a displacement vector $\delta \boldsymbol{\Gamma}$, i.e. the distance between two close points in the phase space, can be written, in the vanishing limit, as

$$
\delta \dot{\boldsymbol{\Gamma}}(t)=\mathbf{T} \cdot \delta \mathbf{\Gamma}
$$

where $\mathbf{T}$ is the Jacobian (or stability) matrix $\mathbf{T} \equiv \partial \dot{\boldsymbol{\Gamma}} / \partial \boldsymbol{\Gamma}$. The solution for the tangent vector is therefore

$$
\delta \boldsymbol{\Gamma}(t)=\mathbf{L}(t) \cdot \delta \boldsymbol{\Gamma}(0) \quad \text { with } \quad \mathbf{L}(t)=\exp _{L}\left(\int_{0}^{t} d s \mathbf{T}(s)\right),
$$

where $\mathbf{L}(t)$ is the propagator and $\exp _{L}$ is the left-ordered exponential. The Lyapunov exponents can be defined as [32]

$$
\lambda^{i}=\lim _{t \rightarrow \infty} \frac{1}{2 t} \ln \left|\mathbf{L}(t) \cdot \mathbf{u}_{i}\right|
$$

where $\mathbf{u}_{i}$ are the eigenvectors of the matrix $\mathbf{L}^{T}(t) \cdot \mathbf{L}(t)$. A more practical definition for the evaluation of Lyapunov exponents using MD simulation is given by [33]

$$
\lambda^{i}=\lim _{t \rightarrow \infty} \lim _{\delta \boldsymbol{\Gamma} \rightarrow 0} \frac{1}{t} \ln \left(\frac{\left|\delta \boldsymbol{\Gamma}^{i}(t)\right|}{\left|\delta \boldsymbol{\Gamma}^{i}(0)\right|}\right),
$$

where $\left|\delta \boldsymbol{\Gamma}^{i}(t)\right|$ is the length of the $i$ th orthogonal displacement vector at time $t$, and $i=1, \ldots, 4$. Benettin et al. [34-36] used this definition to implement a "classic" algorithm that can be adapted to $\mathrm{MD}$, and which is used in this work. A set of 4 displacement vectors $\delta \boldsymbol{\Gamma}_{i}$ is generated at time $t=$ 0 and evolved forward in time with the use of the Jacobian matrix and maintained orthonormal 
performing a Gram-Schmidt procedure at a fixed number of time steps $n \Delta t$. Alternative methods have also been proposed by Hoover and Posch [37] and independently by Goldhirsch et al. [38].

It is worth noting that the multiplicative theorem of Oseledec [39] assures that the matrix

$$
\mathbf{\Lambda}=\lim _{t \rightarrow \infty} \mathbf{\Lambda}(t)=\lim _{t \rightarrow \infty}\left[\mathbf{L}^{T}(t) \cdot \mathbf{L}(t)\right]^{\frac{1}{2 t}},
$$

is well defined in that the limit exists (except for a set of zero measure), but makes no assumptions on the ergodicity of the system. However, if the system is ergodic, the limit is unique and the Lyapunov exponents will not depend on the particular initial condition that we choose for our trajectory. Different values of the Lyapunov exponents for different initial conditions can therefore prove the non-ergodicity of our system.

We would also like to argue that, due to the convex, and therefore defocusing, nature of the collisions between gas and wall particles, the possibility of sub-exponential separation for an ergodic trajectory in phase space should be precluded. Trajectories at the boundaries of elliptic islands could have sub-exponential separation for some time, e.g. a trajectory bouncing against a corner polygon $[15,16]$, however if these trajectories are to be ergodic, they are bound to collide with the convex surface of wall atoms, and this unstable dynamics (i.e. exponential instability) would quickly dominate. A dynamic characterized by sub-exponential growth is said to present "weak-chaos" (e.g. as it can be found in some polygonal billiard systems) which could still give rise to ergodic dynamics [19]. Therefore, in our system, a zero Lyapunov exponent would indicate the presence of periodic orbits and a non chaotic dynamics.

The phenomenon of weak-chaos has attracted wide interest in recent years mainly associated to anomalous diffusion and intermittency particularly in low-dimensional maps [40-42]. These systems are said to give rise to weak ergodicity breaking, referring to the fact that, even though the systems are still ergodic, they can occasionally enter a phase space area where they will spend a large amount of time, so that the ergodic invariant measure is infinite [43], i.e. non-normalisable.

Because we deal with an Hamiltonian system, the Lyapunov exponents will sum to zero [44](i.e. for each positive Lyapunov exponent there must exist an exponent equal in magnitude, but opposite in sign), which also reflects the property of Hamiltonian systems to be phase space volume preserving. We will therefore concentrate our attention only on the largest, and for our purpose most important, of the four Lyapunov exponents.

Every trajectory was followed for a time $t^{*}=2000$ in reduced units. This time was chosen to ensure the convergence of the exponents which, given the small size of the channel, was quickly reached. It is, however, not possible to ensure that the gas particle at some later time $t>t^{*}$ will not wander off what appears to be a periodic orbit and starts to travel the whole phase space. Therefore, as already mentioned in the introduction, with this study we can not prove if the system is or is not ergodic in the infinite time limit, but rather if the onset of ergodic behaviour is evident on time scales accessible to simulations and experimental observations [45].

Our interest lies in measuring the portions of phase space characterized by positive and zero Lyapunov exponents, in order to give a measure for the ergodic and non-ergodic components of phase space respectively. We will use a phase function $B(\boldsymbol{\Gamma})$ to decompose the phase space in two areas, assuming for example the value +1 for areas of positive exponents $\lambda^{+}$, and -1 for areas of zero exponents $\lambda^{0}$, i.e. $\left[B\left(\boldsymbol{\Gamma}^{*}\right)=+1\right.$ if $\left.\lambda\left(\boldsymbol{\Gamma}^{*}\right)>0\right]$ where $\boldsymbol{\Gamma}^{*}$ refers to the starting point of a particular trajectory. We then compute the ensemble average of $B$ and normalize it to generate a measure.

The microcanonical ensemble can be defined in two ways, the shell microcanonical ensemble and the surface microcanonical ensemble[25]. In MD simulations, the phase space trajectory never leaves the surface of constant energy, therefore it seems more natural to use the probability distribution function of the surface microcanonical ensemble

$$
f(\boldsymbol{\Gamma})=\frac{\delta(H(\boldsymbol{\Gamma})-E)}{\int_{\Omega(E)} \delta(H(\boldsymbol{\Gamma})-E) \mathrm{d} \boldsymbol{\Gamma}}=\frac{\frac{1}{\nabla H(\boldsymbol{\Gamma})}}{\int_{S(E)} \frac{1}{\nabla H(\boldsymbol{\Gamma})} \mathrm{d} S_{E}},
$$


where $S(E)$ represents the isosurface and $\nabla H(\boldsymbol{\Gamma})$ is the weight that accounts for the thickness of the energy shell between $E$ and $E+\Delta E$. We can therefore write the ensemble average of an observable as

$$
\langle B\rangle=\int B(\boldsymbol{\Gamma}) f(\boldsymbol{\Gamma}) \mathrm{d} \boldsymbol{\Gamma} \simeq \sum_{i} B\left(\boldsymbol{\Gamma}_{i}\right) w\left(\boldsymbol{\Gamma}_{i}\right),
$$

where $w\left(\boldsymbol{\Gamma}_{i}\right)$ represents a weighting function to be associated to each point $\boldsymbol{\Gamma}_{i}$ in an actual simulation. We need now to appropriately sample the phase space in order to choose the initial conditions for the gas particle. Many methods have been proposed in the past for the sampling of the microcanonical ensemble for molecular systems [46-49], and we used the one by Severin et al. [48], which we only outline briefly. The idea behind the Severin method is to rewrite the distribution function as

$$
f(E \mid \mathbf{\Gamma})=g(E \mid \mathbf{Q}) h(E \mid \mathbf{Q}, \mathbf{P}),
$$

where $\mathbf{Q}$ and $\mathbf{P}$ represent the configurational and momentum space respectively, $g$ is the configurational space probability density and $h$ is the conditional momentum space density given $\mathbf{Q}$. It is easy to show that the configurational density is proportional to the momentum density

$$
g(E \mid \mathbf{Q})=C[E-U(\mathbf{Q})]^{(S / 2-1)}, \quad U(\mathbf{Q})<E,
$$

where $C$ is a constant and $S$ represents the independent momentum coordinates, which in our system are $S=2$. Once a point $\mathbf{Q}$ is chosen with the proper weight, we can proceed to sample the momenta randomly, for example by picking a vector on a circumference of radius $[E-V(\mathbf{Q})]$. In our system $g$ is unity, so no weight is needed. We can picture this maybe surprising result if we think that a longer amount of time spent by the particle close to the walls is counter balanced by a lower momenta density.

The contour plots of Fig. 5 show how the Lyapunov exponents are distributed across the channel $y_{0}=1.1$. They were obtained by sampling $1 \times 10^{6}$ positions and choosing the momenta accordingly. The contour at a particular position in the channel was computed from the exponent value generated by the trajectory starting at that point and corresponding to one random velocity direction. Lyapunov exponents are a property of the trajectory. Ideally, if the system is ergodic the whole phase space is filled by the same trajectory, to which every point will belong, giving as a result the same Lyapunov exponent for all the points in the channel. The contour plots show that some sets of points are associated to different Lyapunov exponents values, and therefore they belong to different trajectories proving that the system is not ergodic. A Lyapunov exponent was regarded to be zero below the threshold value of $\lambda<0.01$. This value was chosen taking into consideration the inaccuracy due to the limited amount of simulation time. Also we note from the histograms in Figs. 6, that the transition between chaotic and non-chaotic regions, i.e. between non-zero and close-to-zero Lyapunov exponents, is sharp, making the identification of a threshold value less arbitrary. In chaotic systems the property of a trajectory to spend long times around the border of stable islands before entering the chaotic sea (i.e. stickiness), can hinder the convergence of dynamical properties. Stickiness is mainly due to the presence of cantori (broken KAM tori) which create a barrier difficult to cross [50,51]. It can delay the convergence of dynamical properties, e.g. Lyapunov exponents, and its strength is a property of the system under consideration. In Figs. 7 we show the convergence of the maximum Lyapunov exponents for 5 trajectories for the channel with $y_{0}=1.1$ and $E=-1.0$. It can be seen that the convergence is nearly instantaneous, with respect to the timescales of interest in this work, demonstrating that stickiness plays little role in our system. Some of the trajectories characterized by zero exponents could require longer time scales to separate, however as pointed out earlier, an experiment could not distinguish between the latter case and one in which the trajectories genuinely belong to stable regular islands.

The contour plots represent only slices of the phase space and do not take into account the momenta space, therefore in Fig.6 we also show the exponents' distribution at 2 randomly chosen 
points in configurational space for $2 \times 10^{4}$ uniformly distributed velocity directions. Figures 5 and 6 complement each other and give us a clear view of a non-ergodic phase space.

In Fig. 8 we show a surface contour plot of the Lyapunov exponents across the channel of width $y_{0}=1.1$. The black lines are the isolines of the potential energy while the red lines the isolines of the Lyapunov exponents value. These were computed at each point in the channel from the trajectory generated by "dropping" the particle in that location without kinetic energy. This means that the Lyapunov exponents belong to different energy shells. Lack of ergodicity in this case can be observed by following an energy isoline and noting that it cuts across different values of Lyapunov exponents, i.e. if the system was ergodic, trajectories on the same energy shell would give the same Lyapunov exponent following what has been discussed earlier. In particular, the protruding periodic island (bordered by the zero valued exponent isoline) in the bottom left corner of the plot is very interesting. Even though the island passes through several energy shells, the size of its width might suggests the presence of focusing collisions (elliptic periodic orbits) in small areas of the channel.

As a final result, in Table 1 we give a normalized measure to the portion of phase space associated with positive and zero exponents for the 4 channels widths $y_{0}$ and the 2 energy shells $E=-1.0$ and $E=+5.0$. The measure of non-ergodic phase space is, if not zero, very small in all cases, suggesting that the assumption of ergodicity for confined pores might be justified. It is worth repeating that these values are not very sensitive from the chosen threshold value of $\lambda$. The sharp transition from chaotic to non-chaotic dynamics could be due to the ease with which trajectories depart from a neighboring elliptic orbit even on these short time scales. We would like to point out that, even though Table 1 may look "scarce", its purpose is to show that while it is possible to have systems interacting via soft potentials, whose geometries admit the existence of stable periodic orbits, their non-ergodic measure is however irrelevant for experimental purposes. The parameters chosen are illustrative of the possible energy landscape and energies one can have in a slit pore. A statistically relevant study of the state points allowed by the system, would also be computationally prohibitive.

\section{Conclusion}

In this study we analyzed the dynamical properties of a system composed of a particle trapped in a nanopore interacting with the confining atomistic walls via a soft potential. We focused our analysis on the timescales typically accessible to experimental observations (e.g. the time to observe diffusion of individual molecules through nanopores in membranes). The results suggest that the ergodic hypothesis is valid for a wide range of energies and channel widths, and even when not strictly valid, the measure of non-ergodic phase space is too small to be of any practical relevance. This result is significant in light of the increasing efforts being focused at the nanoscale, where the ergodic hypothesis is commonly assumed in order to apply statistical mechanics. 
Table 1 Portion of phase space characterized by positive $\left(\lambda^{+}\right)$and zero $\left(\lambda^{0}\right)$ Lyapunov exponents for 4 channel width and 2 energy shell each. We note that the measure of non-ergodic phase space does not seem to follow any particular trend based on the value of the energy and the channel width.

\begin{tabular}{lcrr}
\hline \hline Channel width $y_{0}$ & Energy level & $\lambda^{+}$ & $\lambda^{0}$ \\
\hline 1.1 & -1.0 & 0.97727 & 0.02273 \\
1.1 & +2.0 & 0.99389 & 0.00611 \\
1.1 & +5.0 & 0.99742 & 0.00258 \\
\hline 1.21 & -1.0 & 0.99991 & 0.00009 \\
1.21 & +2.0 & 0.99933 & 0.00067 \\
1.21 & +5.0 & 0.99916 & 0.00084 \\
\hline 1.228 & -1.0 & 1.00000 & 0.00000 \\
1.228 & +2.0 & 0.99928 & 0.00072 \\
1.228 & +5.0 & 0.99969 & 0.00031 \\
\hline 1.4 & -1.0 & 1.00000 & 0.00000 \\
1.4 & +2.0 & 1.00000 & 0.00000 \\
1.4 & +5.0 & 0.99992 & 0.00008 \\
\hline \hline
\end{tabular}


10

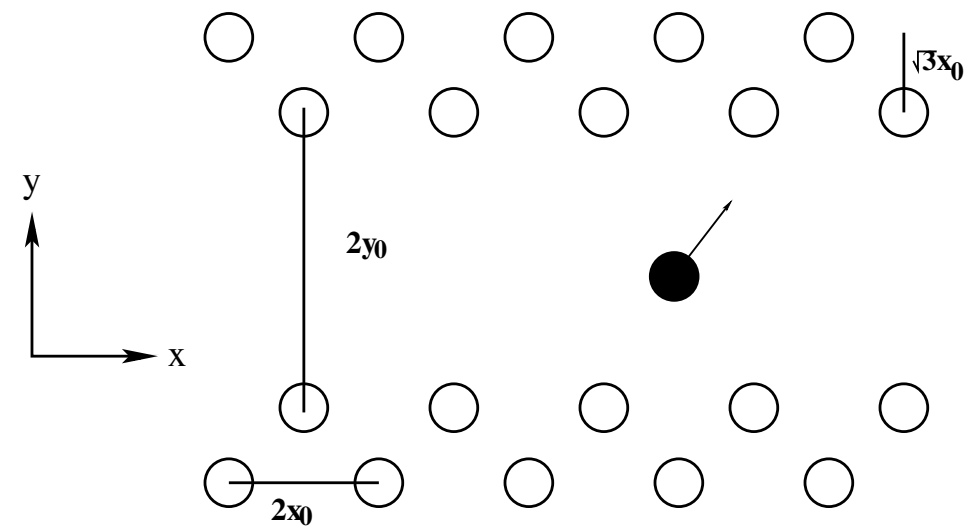

Fig. 1 Schematic illustration of the system. White circles represent the fixed wall atoms and the black circle the mobile particle. $x_{0}$ is a lattice constant with value $x_{0}=0.5557$ and $y_{0}$ is a variable parameter that determines the width of the channel.
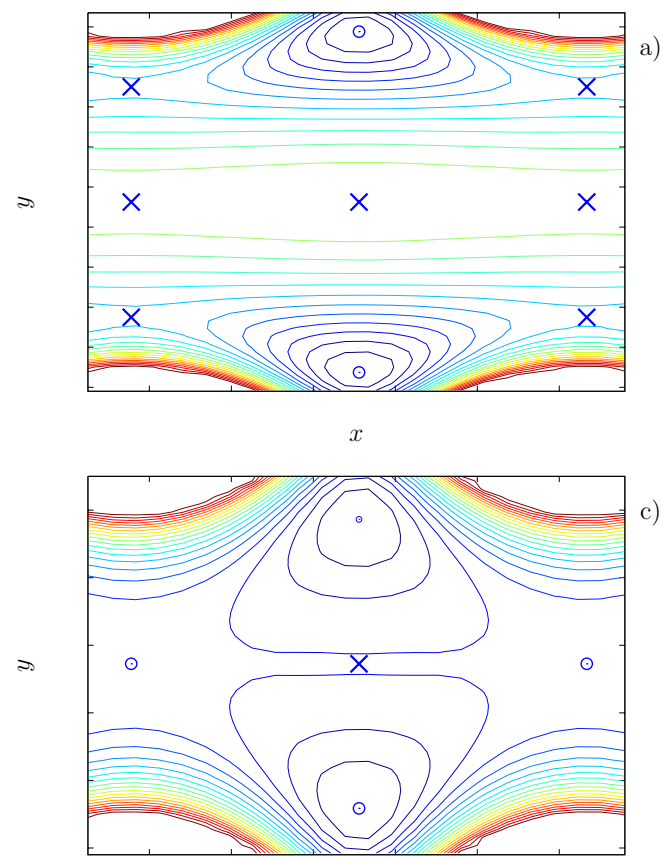

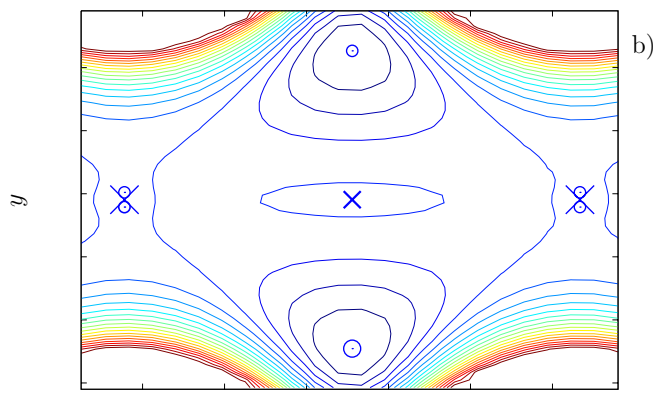

$x$

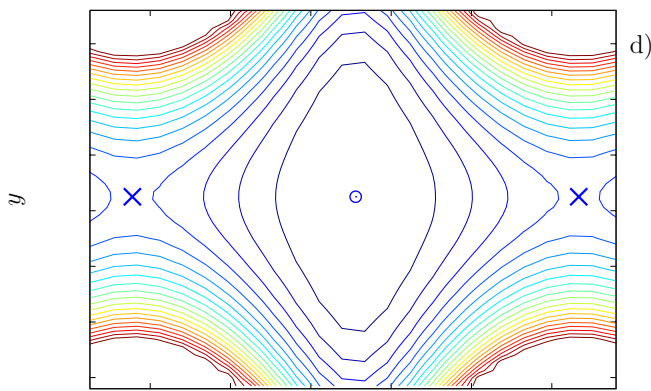

$x$

Fig. 2 Potential energy contour plots in a region of the domain. The superimposed symbols indicate the fixed points in the $(x, y)$ plane: $\times$ saddle point; $\circ$ center point. a) $\left.y_{0}=1.4, \mathrm{~b}\right) y_{0}=1.228$, c) $y_{0}=1.21$, d) $y_{0}=1.1$. Blue contour isolines correspond to lowest potential energy and red to highest potential energy. White regions at the boundaries indicate the areas where the potential energy tends to infinite and goes off-scale. Color online. 


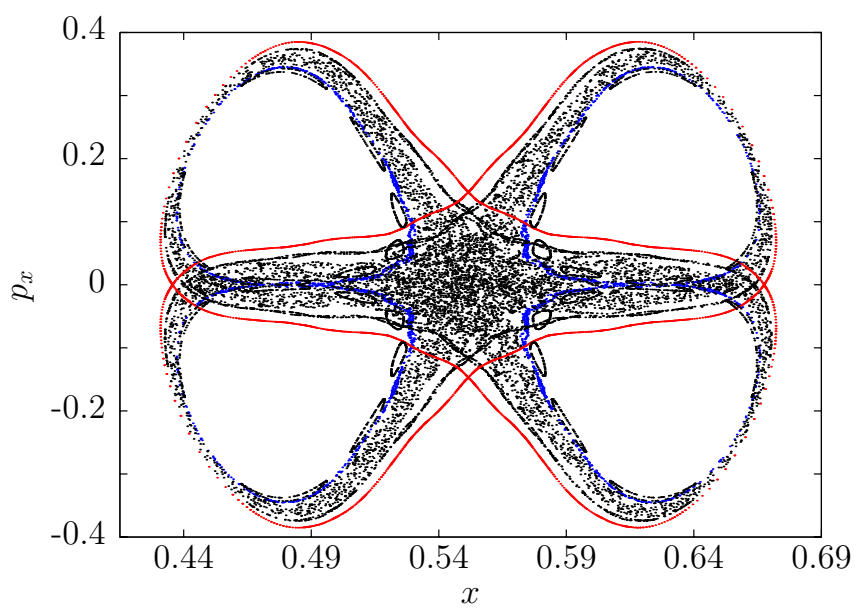

Fig. 3 Poincaré section in the $\left(x, p_{x}\right)$ plane for $y=\sqrt{3} x_{0}+1$ and $y_{0}=2.5$ and $E=-2.0$. To reveal the finer details we have plotted the points with three different colors. Color online.
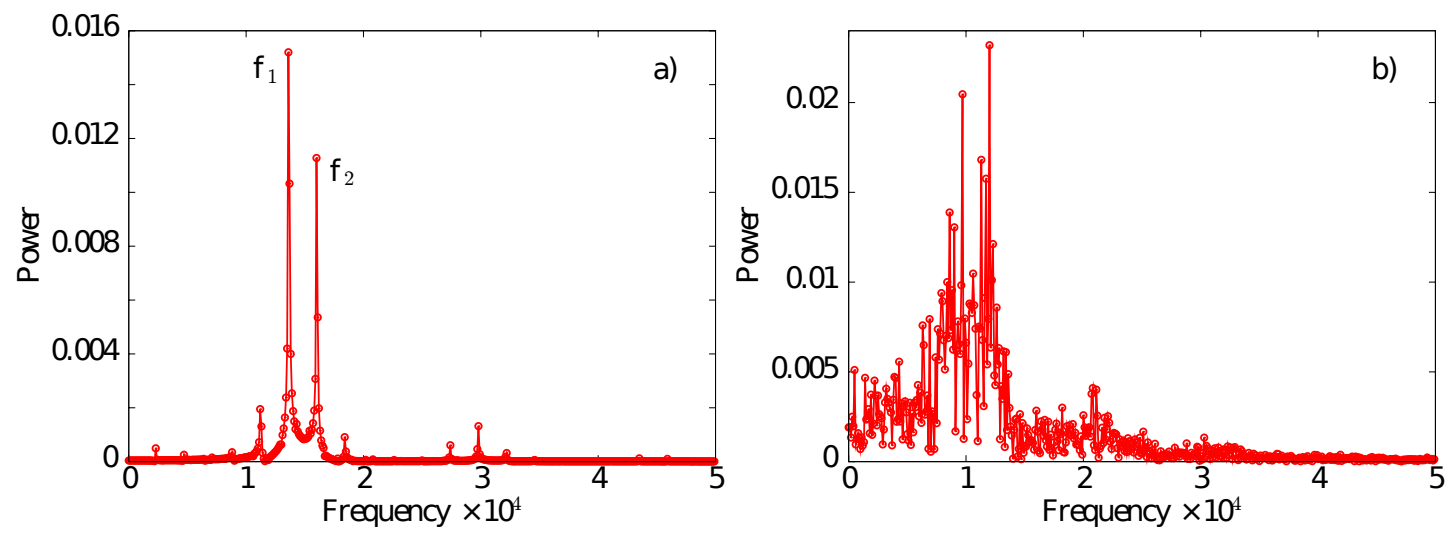

Fig. 4 Power spectra (arbitrary units) for $E=-2$ (a) and $E=-1$ (b) where $y_{0}=2.5$. The two main frequencies for $E=-2$ are given by $f_{1}$ and $f_{2}$. Color online. 
12
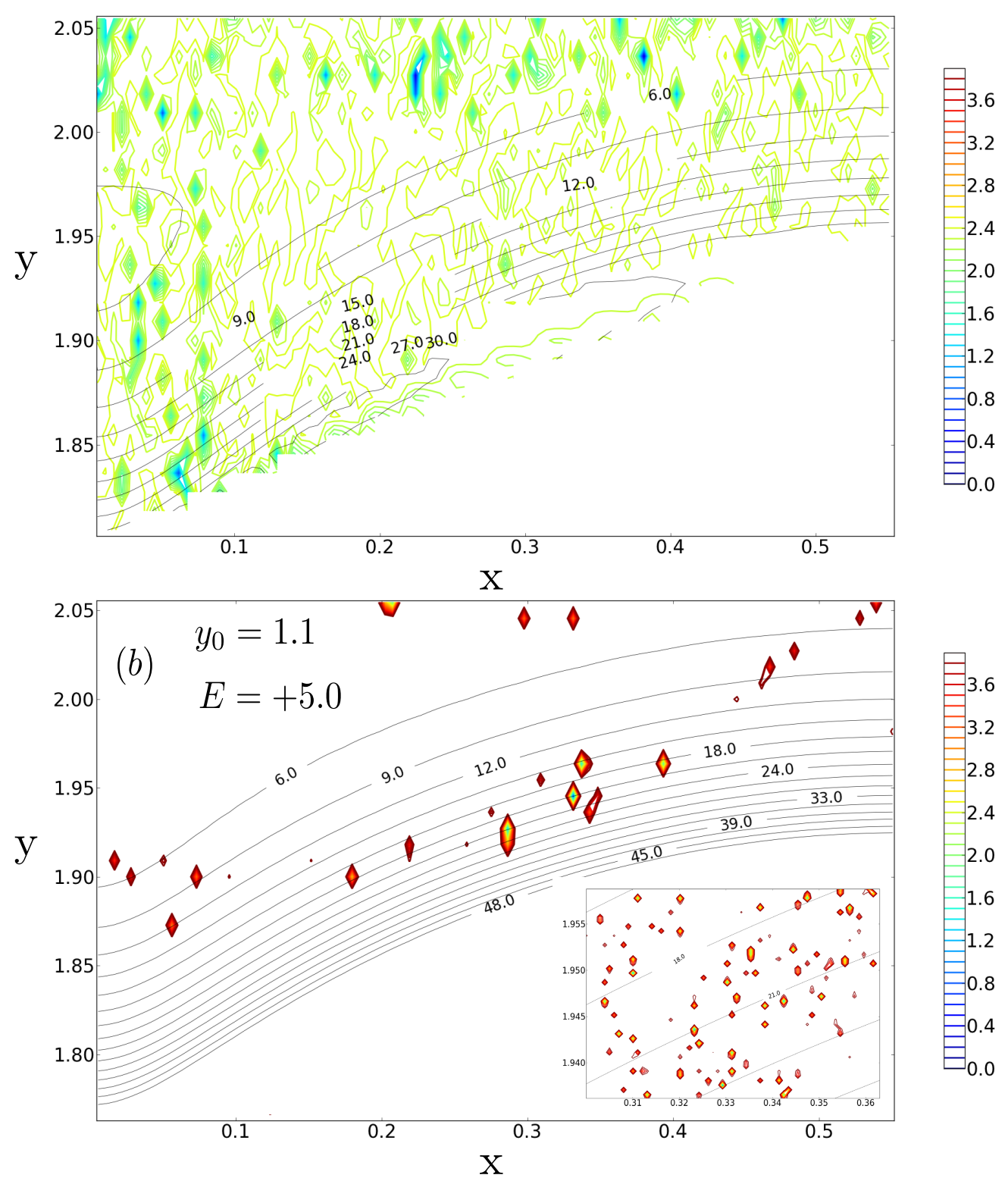

Fig. 5 Surface contour plots of the Lyapunov exponents across the channel of width $y_{0}=1.1$ for 2 values of energy shell $E=-1.0$ and $E=+5.0$. Image obtained with $1 \times 10^{6}$ sampling points. The black lines demarcate isolines of the norm of the Hamiltonian gradient. In Figure b) we also plot an inset to show at a finer detail, the distribution of zero exponents in a small area of the channel (same color gradient as the main plot). Color online. 

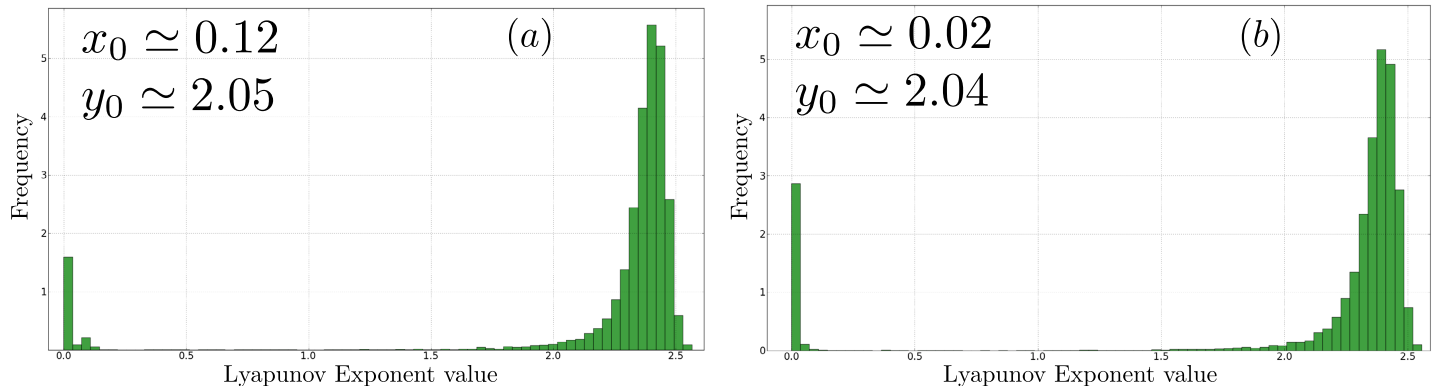

Fig. 6 Histograms of Lyapunov exponent values for $2 \times 10^{4}$ initial momenta direction at 2 randomly chosen initial positions. Channel width $y_{0}=1.1$ and energy shell $E=-1.0$. Color online.

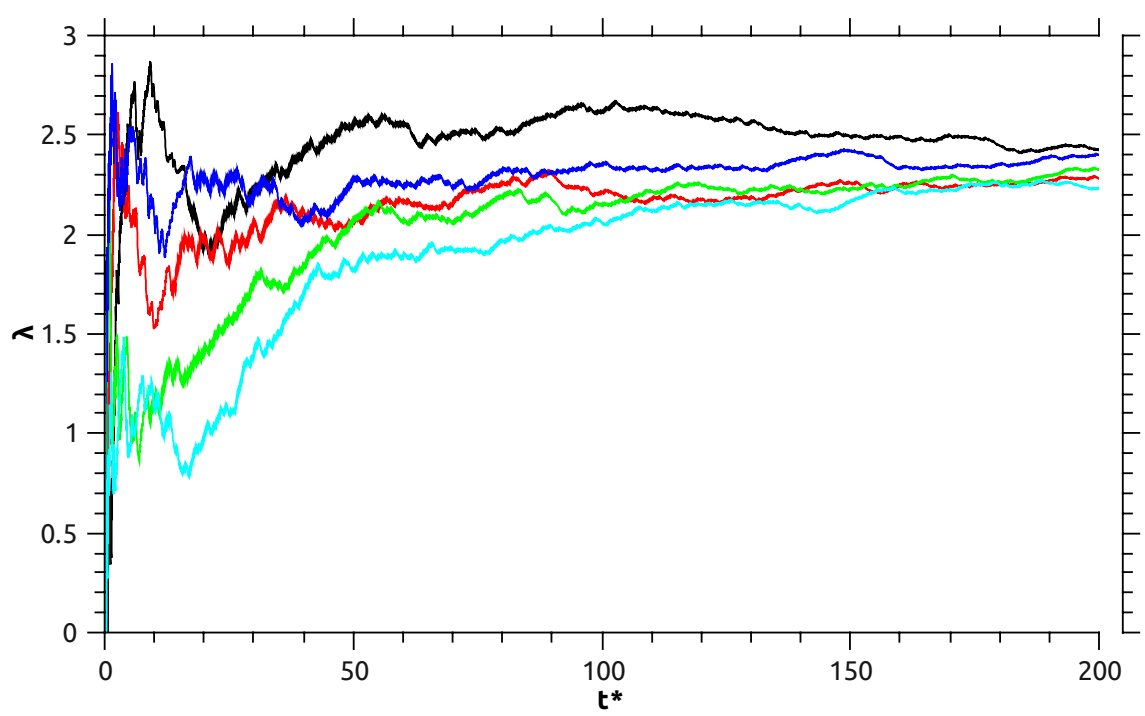

Fig. 7 Convergence over $t^{*}=200$ time units of 5 Lyapunov exponents for 5 different trajectories. Channel's width $y_{0}=1.1$ and energy shell $E=-1.0$. We do not plot more than 5 exponents (relative to one parameter's choice) for presentation clarity. The convergence is nearly instantaneous showing that stickiness does not play a big role in our system. Color online. 
14

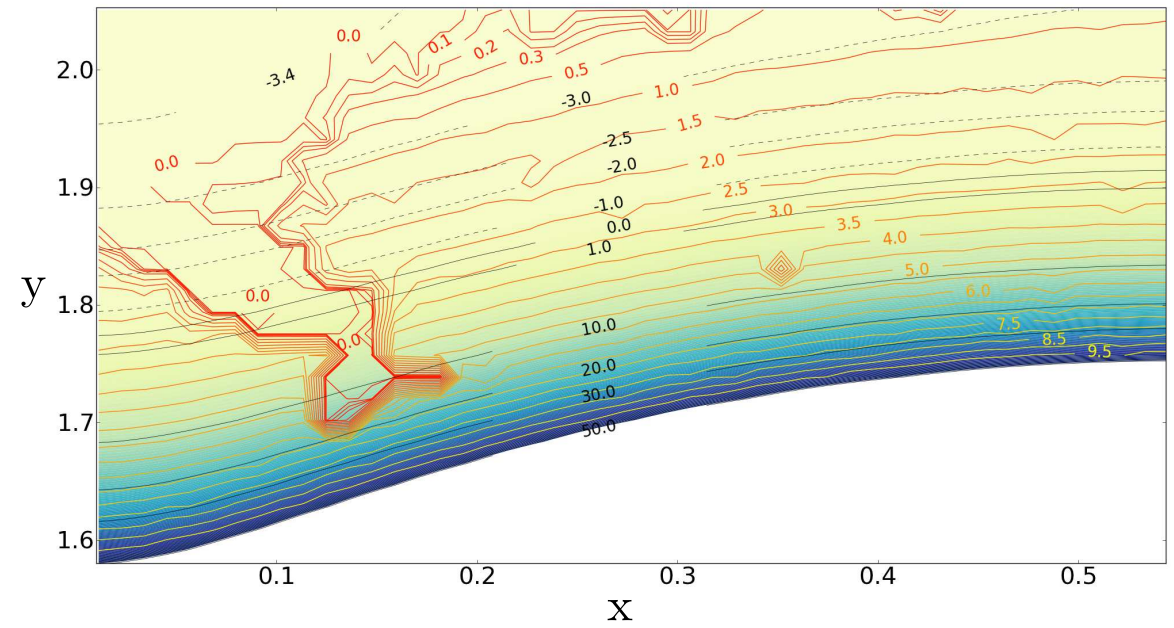

Fig. 8 Surface contour plots of the Lyapunov exponents across the channel of width $y_{0}=1.1$. Image obtained with $1 \times 10^{6}$ sampling points. The black lines demarcate isolines of the potential energy, the red lines the isolines of the Lyapunov exponent values. The particle was "dropped" in the channel without kinetic energy, therefore even though the Lyapunov exponents refer to different energy shells, lack of ergodicity can be seen by following black isolines of same energy which cut across different values of Lyapunov exponents. In particular it is interesting to see a periodic island delimited by the zero valued exponent isoline, protruding towards the bottom left corner of the plot. Color online. 
Acknowledgements J.S. Hansen wishes to acknowledge Lundbeckfonden for supporting this work as a part of grant No. R49-A5634.

\section{References}

1. V.I. Arnold, A. Avez, Ergodic Problems of Classical Mechanics (Benjamin, New York, 1989)

2. A. Katok, B. Hasselblatt, Introduction to the modern theory of dynamical systems (Cambridge University Press, 1995)

3. A. Khinchin, Mathematical Foundations of Statistical Mechanics (Dover, 1949)

4. E. Fermi, Thermodynamics (Dover, 1956)

5. Y. Sinai, N. Chernov, Russ. Math. Surv. 42, 181 (1987)

6. N. Simanyi, Ergod. Th. \& Dynam. Sys. 19, 741 (1999)

7. L.A. Bunimovich, Commun. math. Phys. 65, 295 (1979)

8. L.A. Bunimovich, Y.G. Sinal, Comm. Math. Phys. 78, 479 (1980)

9. B. Moran, W. Hoover, J. Stat. Phys. 48, 709 (1987)

10. S. Troubetzkoy, J. Stat. Phys. 141, 60 (2010)

11. V.J. Donnay, Ergod. Th. \& Dynam. Sys. 16, 975 (1996)

12. N. Berglund, H. Kunz, J. Stat. Phys. 83, 81 (1996)

13. V.J. Donnay, J. Stat. Phys. 96, 1021 (2001)

14. V.I. Arnold, Mathematical Methods of Classical Mechanics (Springer, New York, 1999)

15. A. Rapoport, V. Rom-Kedar, Chaos 16, 043108 (2006)

16. A. Rapoport, V. Rom-Kedar, Phys. Rev. E 77, 016207 (2008)

17. C. Dettmann, E. Cohen, J. Stat. Phys. 101, 28 (2000)

18. F. Cecconi, M. Cencini, A. Vulpiani, J. Stat. Mech. (2007)

19. O. Jepps, L. Rondoni, J. Phys. A: Math. Gen. 39, 1311 (2006)

20. W. Lan, D.A. Holden, B. Zhang, H.S. White, Anal. Chem. 83, 3840 (2011)

21. W. Lan, H.S. White, ACS Nano (2012)

22. A. Eren, I. Amin, A. Alba, E. Morales, A. Stoyanov, S. Winters-Hilt, Accepted paper in book "Advances in Computational Biology", Springer: Advances in Experimental Medicine and Biology (2010)

23. Y.G. Sinai, Russ. Math. Surv. 25, 137 (1970)

24. H. Goldstein, C. Poole, J. Safko, Classical Mechanics (Addison Wesley, San Francisco, 2002)

25. D.A. Mcquarrie, Statistical Mechanics (Harper and Row, New York, 1976)

26. E. Ott, Chaos in Dynamical Systems (Cambridge University Press, 2002)

27. D. Frenkel, B. Smit, Understanding Molecular Simulation (Academic Press, London, 1996)

28. J. Galán-Vioque, A. Vanderbauwhede, in Numerical Continuation Methods for Dynamical Systems, ed. by S. Verlag (Springer Netherlands, New York, 2007)

29. S. Wiggins, Introduction to Applied Nonlinear Dynamical Systems and Chaos (Springer-Verlag, New York, 1990)

30. S. Bernardi, B. Todd, D. Searles, J. Chem. Phys. 132, 244706 (2010)

31. S. Bernardi, B. Todd, J. Hansen, D. Searles, F. Frascoli, J. Chem. Phys. 132, 244508 (2010)

32. J.P. Eckmann, D. Ruelle, Rev. Mod. Phys. 57(3), 617 (1985)

33. S. Sarman, D.J. Evans, G.P. Morriss, Phys. Rev. A 45(4), 2233 (1992)

34. G. Benettin, L. Galgani, A. Giorgilli, J.M. Strelcyn, Meccanica 15, 9 (1980)

35. G. Benettin, L. Galgani, A. Giorgilli, J.M. Strelcyn, Meccanica 15, 21 (1980)

36. I. Shimada, T. Nagashima, Prog. Theor. Phys. 61, 1605 (1979)

37. W.G. Hoover, H.A. Posch, Phys. Lett. A 113(2), 82 (1985)

38. I. Goldhirsch, P.L. Sulem, S.A. Orszag, Phys. D 27(3), 311 (1987)

39. V. Oseledec, Trudy Mosk. Mat. Obsc. 19, 179 (1968)

40. G. Bel, E. Barkai, Europhys. Lett. 74, 15 (2006)

41. S. Burov, J.H. Jeon, R. Metzler, E. Barkai, Phys. Chem. Chem. Phys. 13, 1800 (2011)

42. N. Korabel, R. Klages, A.V. Chechkin, I.M. Sokolov, V.Y. Gonchar, Phys. Rev. E 75, 036213 (2007)

43. T. Akimoto, Y. Aizawa, Chaos 20, 033110 (2010)

44. C.P. Dettmann, G.P. Morriss, Phys. Rev. E 53(6), 5545 (1996)

45. J. Dorfman, An Introduction to Chaos in Nonequilibrium Statistical Mechanics (Cambridge University Press, 2001)

46. J. Randrup, Comp. Phys. Comm. 59, 439 (1990)

47. D. Bunker, J. Chem. Phys. 37, 393 (1962)

48. E. Severin, B. Freasier, N. Hamer, D. Jolly, S. Nordholm, Chemical Physics Letters 57, 117 (1978)

49. J.R. F.M.S.S. Fernandes, Comp. Phys. Comm. 90, 73 (1995)

50. Y. Zou, M. Thiel, M. Romano, J. Kurths, Chaos 17, 043101 (2007)

51. C. Efthymiopoulos, G. Contopoulos, N. Voglis, R. Dvorak, J. Phys. A: Math. and Gen. 30, 8167 (1997) 


\section{University Library}

\section{- M M I E R R V A gateway to Melbourne's research publications}

Minerva Access is the Institutional Repository of The University of Melbourne

Author/s:

Bernardi, S;Hansen, JS;Frascoli, F;Todd, BD;Dettmann, CP

Title:

Ergodicity of a Single Particle Confined in a Nanopore

Date:

2012-09-01

Citation:

Bernardi, S., Hansen, J. S., Frascoli, F., Todd, B. D. \& Dettmann, C. P. (2012). Ergodicity of a Single Particle Confined in a Nanopore. JOURNAL OF STATISTICAL PHYSICS, 148 (6), pp.1156-1169. https://doi.org/10.1007/s10955-012-0577-8.

Persistent Link:

http://hdl.handle.net/11343/283083 